\title{
Correction to: \\ NKX2-1 Activation by SMADS Signaling After Definitive Endoderm Differentiation in Human Embryonic Stem Cell by Li YU, Eggermont K, Vanslembrouch V, and Verfaillie CM. Stem Cells Dev 2013;22;9:1433-1442 DOI:10.1089/scd.2012-0620
}

7 He Authors of THE ARTICLE entitled, NKX2-1 Activation by SMADS Signaling After Definitive Endoderm Differentiation 1 in Human Embryonic Stem Cell, published in Stem Cells Dev 2013;22;9:1433-1442 wish to correct errors that appeared in Figure 3.

\section{Original Figure 3}

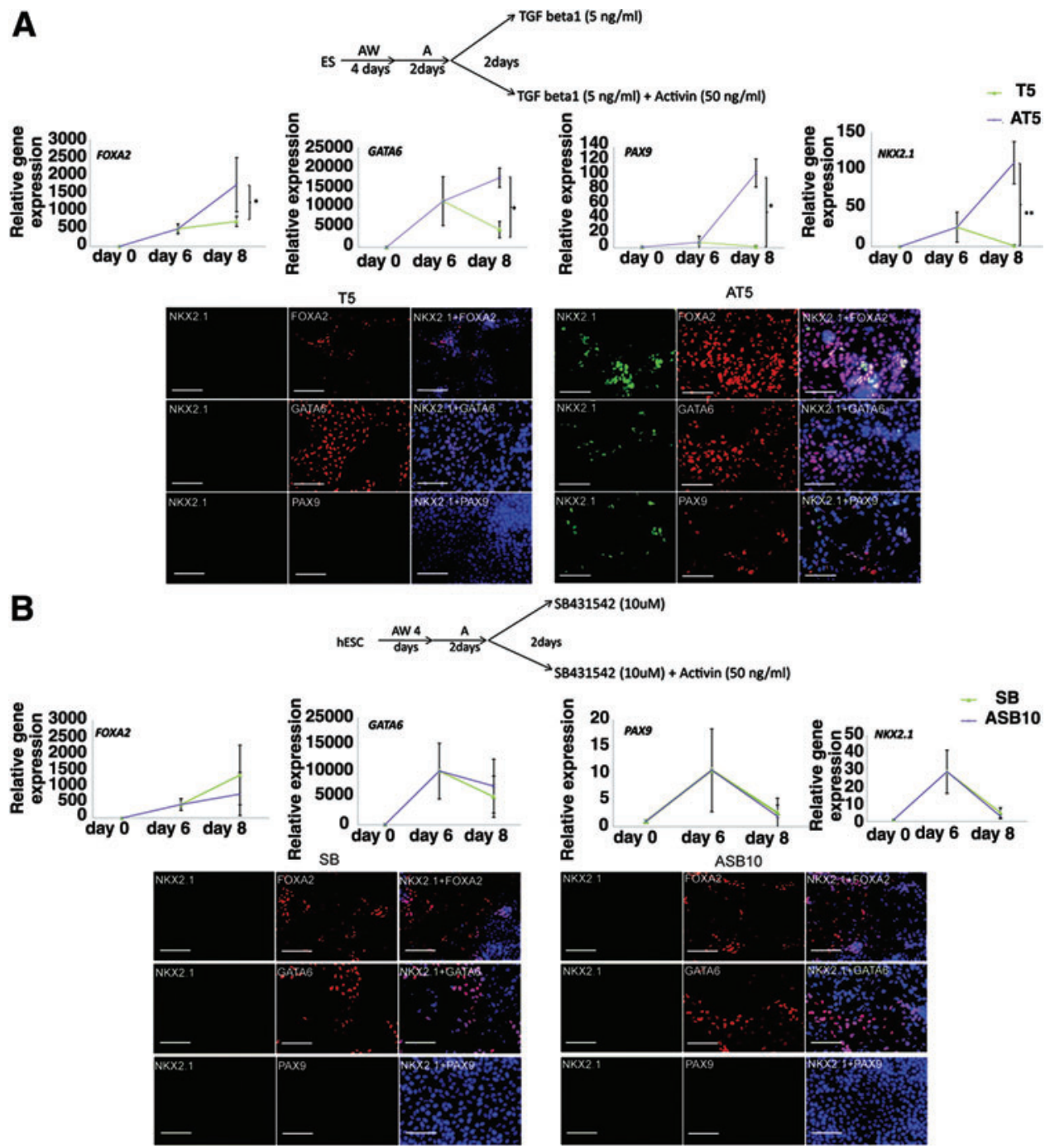

FIG. 3. Induction of NKX2-1 expression is Activin-A specific. (A) RT-qPCR measurement and immunostaining for NKX2-1, FOXA2, GATA6, and PAX9 of $\mathrm{hESC}-\mathrm{H} 9$ cells treated with transforming growth factor (TGF) $\beta 1$ and TGF $\beta 1+$ Activin-A between $\mathrm{d} 6$ and $\mathrm{d} 8$. Data are represented as mean \pm SEM from 5 independent experiments $(* P<0.05 ; * * P<0.01)$. Scale bar, $100 \mu \mathrm{m}$. Percentage FOXA2 positive cells: T5 $=72.3 \% \pm 8.4 \%$, AT5 $=79.5 \% \pm 11.8 \%$; Percentage NKX2-1 positive cells: T5 $=0 \%$; AT5 $=17 \% \pm 11.6 \%(n=3)$. (B) RT-qPCR measurement and immunostaining for NKX2-1, FOXA2, GATA6 and PAX9 of hESC-H9 cells treated with SB431542 and SB431542+Activin-A between d6 and d8. Data are represented as mean \pm SEM from 5 independent experiments. Scale bar, $100 \mu \mathrm{m}$. Percentage FOXA2 positive cells: $\mathrm{SB}=78.1 \% \pm 5 \%, \mathrm{ASB}=86.9 \% \pm 7.9 \%$; Percentage NKX2-1 positive cells $=0 \%(n=2)$. Color images available online at www.liebertpub.com/scd 
There is an incorrect entry in the images in Figure 3, SB431542 treated cells stained for NKX2.1 and FOXA2, which show a partial overlap with images in the top row of the SB431542+Activin-A panel, also stained for NKX2.1 and FOX A2.

The images used in Figure 3 were taken from random images acquired from the same well treated with SB431542+Activin-A with possible partial overlap rather than a well treated with SB431542 and a well treated with SB431542+Activin-A.

\section{The authors have supplied a revised Figure 3 as shown next.}
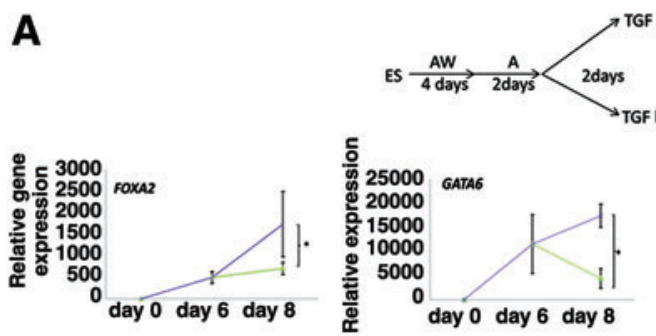

T5
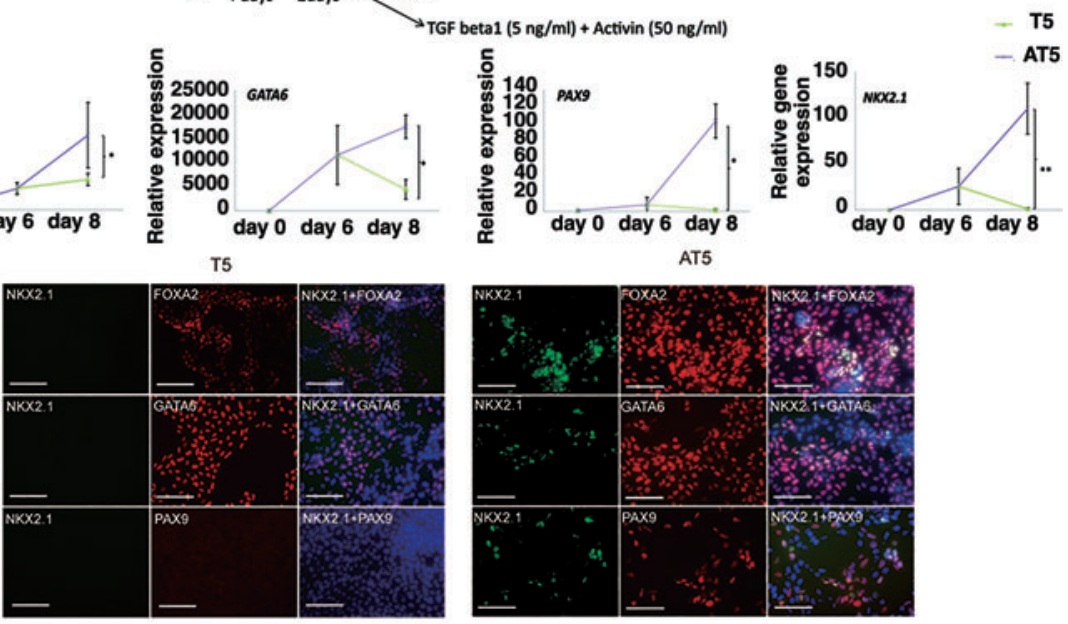

AT5
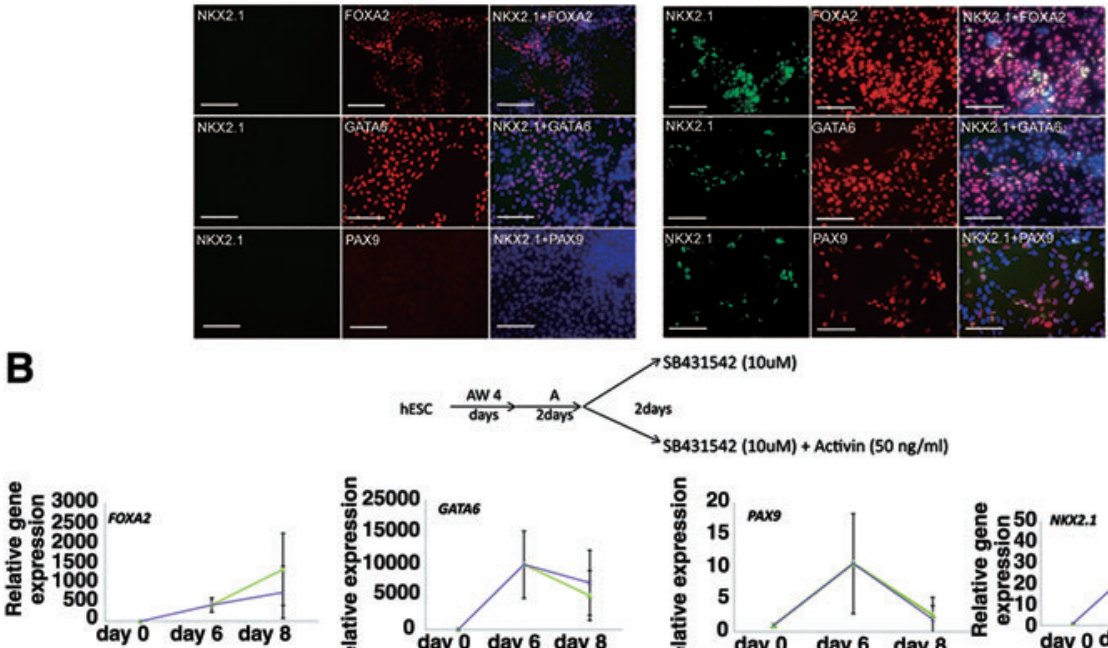

SB431542 (10uM) + Activin $(50 \mathrm{ng} / \mathrm{ml})$
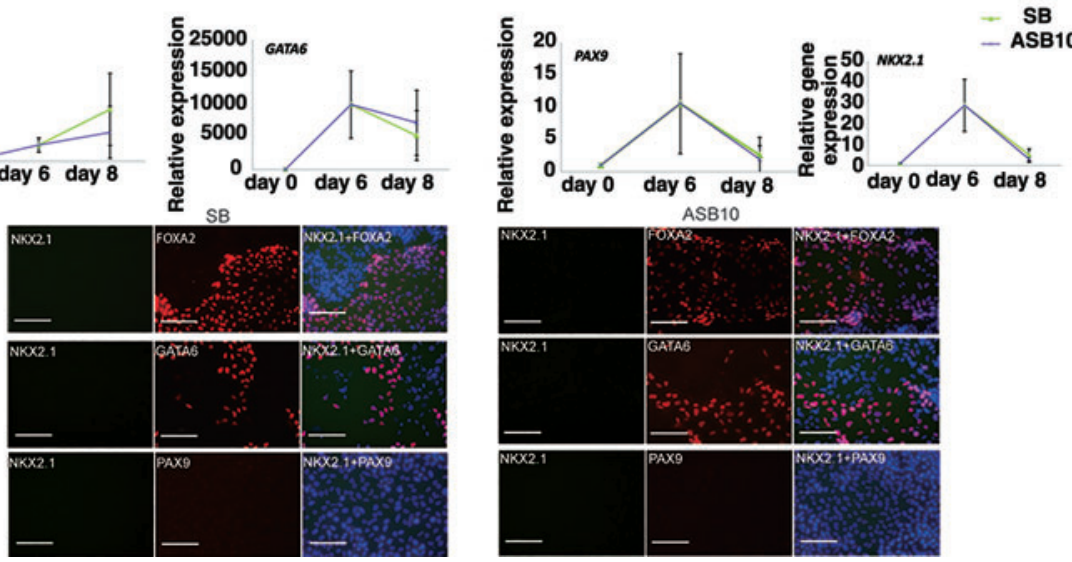

FIG. 3. Induction of NKX2-1 expression is Activin-A specific. (A) RT-qPCR measurement and immunostaining for NKX2-1, FOXA2, GATA6, and PAX9 of hESC-H9 cells treated with transforming growth factor (TGF) $\beta 1$ and TGF $\beta 1+$ Activin-A between d6 and d8. Data are represented as mean \pm SEM from 5 independent experiments $(* P<0.05 ; * * P<0.01)$. Scale bar, $100 \mu \mathrm{m}$. Percentage FOXA2 positive cells: T5 $=72.3 \% \pm 8.4 \%$, AT5 $=79.5 \% \pm 11.8 \%$; Percentage NKX2-1 positive cells: T5 =0\%; AT5 $=17 \% \pm 11.6 \%(n=3)$. (B) RT-qPCR measurement and immunostaining for NKX2-1, FOXA2, GATA6 and PAX9 of hESC-H9 cells treated with SB431542 and SB431542+Activin-A between d6 and d8. Data are represented as mean \pm SEM from 5 independent experiments. Scale bar, $100 \mu \mathrm{m}$. Percentage FOXA2 positive cells: $\mathrm{SB}=78.1 \% \pm 5 \%$, ASB $=86.9 \% \pm 7.9 \%$; Percentage NKX2-1 positive cells $=0 \%(n=2)$. Color images available online at www.liebertpub.com/scd

The online version of this article has been revised to reflect these corrections.

The authors apologize for the mistake; the erroneous image insert did not affect the ultimate conclusion of the manuscript. 